\title{
Short-distance regularity of Green's function and UV divergences in entanglement entropy
}

\author{
Dmitry Nesterov ${ }^{a, b}$ and Sergey N. Solodukhin ${ }^{a}$ \\ ${ }^{a}$ Laboratoire de Mathématiques et Physique Théorique, \\ Université François-Rabelais Tours Fédération Denis Poisson - CNRS, \\ Parc de Grandmont, 37200 Tours, France \\ ${ }^{b}$ Theory Department, Lebedev Physical Institute, \\ Leninsky Prospect 53, 119991 Moscow, Russia \\ E-mail: Dmitry.Nesterov@lmpt.univ-tours.fr, \\ Sergey.Solodukhin@lmpt .univ-tours.fr
}

ABSTRACT: Reformulating our recent result (arXiv:1007.1246 [hep-th]) in coordinate space we point out that no matter how regular is short-distance behavior of Green's function the entanglement entropy in the corresponding quantum field theory is always UV divergent. In particular, we discuss a recent example by Padmanabhan (arXiv:1007.5066 [gr-qc]) of a regular Green's function and show that provided this function arises in a field theory the entanglement entropy in this theory is UV divergent and calculate the leading divergent term.

Keywords: Field Theories in Higher Dimensions, Renormalization Regularization and Renormalons

ARXIV EPRINT: 1008.0777 


\section{Contents}

1 Introduction $\quad 1$

2 Heat kernel, Green's function and entanglement entropy 2

3 Padmanabhan's example $\quad 4$

4 Conclusions 5

\section{Introduction}

Entanglement entropy $[1,2]$ remains a fascinating subject of current research (for a recent review see [3]). It is defined by tracing degrees of freedom residing inside a surface $\Sigma$ and, to some degree, measures the short-distance correlations across the surface. Its geometrical feature (the proportionality to the area $A$ of the surface) makes it a very attractive candidate to provide a statistical explication to the gravitational entropy associated to horizons. A major difficulty on this way, however, is the fact that entanglement entropy calculated for a quantum free field is UV divergent. In $d$ space-time dimensions one has that

$$
S_{\text {ent }} \sim \frac{A}{\epsilon^{d-2}}
$$

where $\epsilon$ is an UV cut-off. A simple (and perhaps somewhat naive) way to understand the origin of the divergence in the entropy is to relate it to the short-distance divergence of the 2-point function, in $d$ space-time dimensions one has in a standard field theory

$$
<\phi(x), \phi(y)>=G(x, y)=\frac{\Omega_{d}}{|x-y|^{d-2}},
$$

where $\Omega_{d}=\frac{\Gamma\left(\frac{d-2}{2}\right)}{4 \pi^{\frac{d}{2}}}$ and $G(x, y)$ is Green's function. At first sight this relation seems natural: two sub-systems separated by surface $\Sigma$ know about each other due to the short-distance correlations that exist between the modes residing on different sides of the surface. As a result of this correlation the entropy is non-vanishing and is determined by geometry of the surface, to leading order by the area. Since the short-distance correlations are divergent, as in (1.2), this divergence seems to manifest in the UV divergences in the entropy (1.1).

Therefore, one may think that there is a one-to-one correspondence between divergences in (1.1) and (1.2) so that one may expect that in a theory in which 2-point functions are regular in the coincidence limit the entanglement entropy would be automatically UV finite. A simple example of this sort is the following modification of (1.2)

$$
G_{L}(x, y)=\frac{\Omega_{d}}{\left((x-y)^{2}+L^{2}\right)^{\frac{d-2}{2}}}
$$


where the short-distance divergences are now regularized by parameter $L$. This example was recently considered by Padmanabhan [4] who argued that in a theory with Green's function (1.3) entanglement entropy is UV finite. In his approach the parameter $L$ incorporates some fundamental, possibly stringy, effects so that $G_{L}$, as it appears in [4], is seemingly not a field theoretical Green's function. Nevertheless, since (1.3) may well appear in some field theory, one may have impression from reading [4] that in the field theory the regularity of Green's function in the coincidence limit implies the UV finiteness of entanglement entropy. The latter is not the case as we show in this note.

In a recent paper [5] we have proved a sort of "no-go theorem" by showing that no matter how well is UV behavior of the propagator of a quantum field theory the entanglement entropy calculated in this theory is always UV divergent. We have showed this by using momentum representation of the heat kernel for a quantum field satisfying a rather general (Lorentz invariant or non-Lorentz invariant) field equation. Obviously a field theory, in which (1.3) appears as Green's function, belongs to the class of theories we have considered and hence entanglement entropy in this theory is still UV divergent even though the short-distance correlations in (1.3) are regular.

In this note we first reformulate the statement made in [5] in terms of Green's function and then discuss a theory with Green's function of the type (1.3). For simplicity we only consider Lorentz invariant field theories.

\section{Heat kernel, Green's function and entanglement entropy}

We consider a quantum field that satisfies a rather general Lorentz invariant field equation

$$
\mathcal{D} \phi=F(\square) \phi=0,
$$

where $F(\square)$ is an arbitrary function of the Laplace operator $\square=-\partial_{\mu} \partial^{\mu}$. Many important quantities that characterize the quantum field can be expressed in terms of the heat kernel $K\left(s, X, X^{\prime}\right)=<X\left|e^{-s \mathcal{D}}\right| X^{\prime}>$ (for a standard review on the heat kernel method see [6]). The latter is defined as a solution to the heat equation

$$
\left\{\begin{array}{l}
\left(\partial_{s}+\mathcal{D}\right) K(s, X, Y)=0, \\
K(s=0, X, Y)=\delta(X, Y) .
\end{array}\right.
$$

In particular, the effective action is defined as

$$
W=-\frac{1}{2} \int_{\epsilon^{2}}^{\infty} \frac{d s}{s} \operatorname{Tr} K(s),
$$

where parameter $\epsilon$ is an UV cutoff. In flat spacetime one can use the Fourier transform in order to solve the heat equation (2.2). In $d$ spacetime dimensions one has

$$
K(s, X, Y)=\frac{1}{(2 \pi)^{d}} \int d^{d} p e^{i p_{\mu}\left(X^{\mu}-Y^{\mu}\right)} e^{-s F\left(p^{2}\right)} .
$$

Note that we consider Euclidean theory so that $p^{2} \geq 0$. The Green's function is a solution to the field equation with a delta-like source

$$
\mathcal{D} G(X, Y)=\delta(X, Y)
$$


and can be expressed in terms of the heat kernel as follows

$$
G(X, Y)=\int_{0}^{\infty} d s K(s, X, Y) .
$$

Obviously, Green's function can be represented in terms of the Fourier transform in a manner similar to $(2.4)$,

$$
G(X, Y)=\frac{1}{(2 \pi)^{d}} \int d^{d} p e^{i p_{\mu}\left(X^{\mu}-Y^{\mu}\right)} G\left(p^{2}\right) .
$$

Using (2.6) or Fourier transformed (2.5) we find that

$$
G\left(p^{2}\right)=1 / F\left(p^{2}\right) .
$$

In a Lorentz invariant theory described by equation (2.1) Green's function (2.7) is a function of the space-time interval $\sigma=(X-Y)^{2}$. On the other hand, any given Green's function $G(\sigma)$ can be Fourier decomposed as in (2.7). Then using relation (2.8) we can restore the field equation this Green's function satisfies. In the coincidence limit, $X=Y$, we find from $(2.7)$

$$
G(X, X)=\frac{2}{\Gamma\left(\frac{d}{2}\right)} \frac{1}{(4 \pi)^{\frac{d}{2}}} \int_{0}^{\infty} d p p^{d-1} G\left(p^{2}\right) .
$$

This limit is finite if function $G\left(p^{2}\right)$ is decaying faster than $1 / p^{d}$ for large $p$.

As was shown in [5] in a theory described by the field equation (2.1) entanglement entropy takes the form (for simplicity we take $\Sigma$ to be a $(d-2)$-dimensional plane)

$$
S=\frac{A(\Sigma)}{12 \cdot(4 \pi)^{(d-2) / 2}} \int_{\epsilon^{2}}^{\infty} \frac{d s}{s} P_{d-2}(s),
$$

where function $P_{n}(s)$ is defined as follows

$$
P_{n}(s)=\frac{2}{\Gamma\left(\frac{n}{2}\right)} \int_{0}^{\infty} d p p^{n-1} e^{-s F\left(p^{2}\right)} .
$$

Clearly, the function $P_{d-2}(s)$ is divergent in the limit $s \rightarrow 0$ for any function $F\left(p^{2}\right)$ growing at large $p{ }^{1}$ This can be seen by taking literally this limit in the integral $(2.11)$ the integration over $p$ then is divergent in the upper limit. For small but finite $s$ this divergence is translated into divergence in variable $s$. Since arbitrary $F\left(p^{2}\right)$ means arbitrary Green's functions (2.7), including those which are regular at $X=Y$ (i.e. integral in (2.9) is finite), we conclude that no matter how well Green's function behaves in the coincidence limit the entanglement entropy remains UV divergent. This is a slightly different formulation of the statement made in [5].

\footnotetext{
${ }^{1}$ Our consideration is valid for the class of functions $F\left(p^{2}\right)$ for which integral representation (2.4) for the heat kernel is well defined. This class includes functions unboundedly growing for large $p$. One may suggest to interchange the integrations over $p$ and $s$ in (2.10) and (2.11). Then naivley the integral over momenta $\int_{0}^{\infty} d p p^{d-3} \ln F\left(p^{2}\right)$ may seem to be finite if $\ln F\left(p^{2}\right)$ is smaller than $1 / p^{d-2}$. However, this function $F\left(p^{2}\right)$ does not belong to the class of functions specified above and should be excluded from consideration. In fact the integral (2.11) is divergent in this case and hence the interchange of integration over $s$ and $p$ is not allowed. We thank the referee for bringing to us this example.
} 


\section{Padmanabhan's example}

For simplicity in this section we consider the case of space-time dimension $d=4$. Green's function (1.3) gives us an example of Green's function which is regular in coincidence limit $X=Y$. As was noted in a recent paper [4] function (1.3) can be represented in a form similar to $(2.6)$

$$
\begin{aligned}
G_{L}(X, Y) & =\int_{0}^{\infty} d s H(s, X, Y) \\
H(s, X, Y) & =\frac{1}{(4 \pi s)^{2}} e^{-|X-Y|^{2} / 4 s-L^{2} / 4 s} .
\end{aligned}
$$

If function $H(s, X, Y)$ was a heat kernel then the $L$-dependent term in (3.1) would regularize all UV divergences so that the effective action (2.3) and entanglement entropy (2.10) would appear UV finite. This is the point of view advocated in [4]. However, despite the apparent similarity of (3.1) and (2.6) the function $H(s, X, Y)$ is NOT a heat kernel: it does not satisfy neither the field equation (2.2) for any differential operator $\mathcal{D}$ nor the "initial condition" at $s=0$, i.e. it does not reproduce a delta-function.

On the other hand, the Fourier transform for Green's function $G_{L}(X, Y)(1.3)$ is well defined. We find that $[7]$

$$
G\left(p^{2}\right)=\frac{L}{p} K_{1}(p L)
$$

The corresponding heat kernel is defined by (2.4) taking into account relation (2.8). Hence we can reconstruct the relevant field equation $F(\square) \phi=0$ and obtain

$$
F(\square)=\frac{\sqrt{\square}}{L} \frac{1}{K_{1}(L \sqrt{\square})} .
$$

For small values of $p$ function $F\left(p^{2}\right)=G^{-1}\left(p^{2}\right)$ behaves as $p^{2}$ while for large $p$ it grows exponentially

$$
F\left(p^{2}\right) \simeq \sqrt{\frac{2}{\pi L}} p^{3 / 2} e^{p L} .
$$

For a function $F\left(p^{2}\right)$ with asymptotic behavior (3.4) we have that

$$
P_{2}(s)=\frac{1}{L^{2}}\left(\ln \frac{s}{L^{2}}\right)^{2}+O\left(\ln \frac{s}{L^{2}}\right) .
$$

Hence entanglement entropy (2.10) is UV divergent and the leading divergence

$$
S=\frac{A}{48 \pi L^{2}} \frac{1}{3}\left(\ln \frac{\epsilon^{2}}{L^{2}}\right)^{3}
$$

is logarithmic. This is despite the fact that Green's function (1.3) is completely regular in the coincidence limit. 


\section{Conclusions}

In this note we have emphasized that, perhaps contrary to intuition, the short distance regularity of Green's function does not imply the UV finiteness of entanglement entropy. In fact our statement is rather general: in any theory characterized by Lorentz invariant Green's function the corresponding entanglement entropy is UV divergent, the degree of divergence is determined by the behavior of Fourier transform $G\left(p^{2}\right)$ (propagator) for large values of $p$. The requirement of the Lorentz symmetry is not essential. As we show in [5] entanglement entropy remains UV divergent even if the Lorentz symmetry is violated by a generic term in the field operator.

Comparing our results with approach of Padmanabhan [4] we should note that from the field theoretical point of view his prescription to use the function $H(s, X, Y)$ instead of heat kernel can be viewed as a new method of regularization of the UV divergences similar to the Pauli-Villars regularization. In his regularization parameter $L$ plays the role of the UV cut-off similar to the parameter $\epsilon$ in the proper time regularization which we use. Then the divergences in $\epsilon$ of effective action or entropy are just replaced by equivalent divergences when $L$ is taken to zero.

The postdoctoral position of D.N. is financed by the University of Tours. D.N. was also supported by the RFBR grant 08-02-00725.

Open Access. This article is distributed under the terms of the Creative Commons Attribution Noncommercial License which permits any noncommercial use, distribution, and reproduction in any medium, provided the original author(s) and source are credited.

\section{References}

[1] L. Bombelli, R.K. Koul, J.H. Lee and R.D. Sorkin, A quantum source of entropy for black holes, Phys. Rev. D 34 (1986) 373 [SPIRES].

[2] M. Srednicki, Entropy and area, Phys. Rev. Lett. 71 (1993) 666 [hep-th/9303048] [SPIRES].

[3] H. Casini and M. Huerta, Entanglement entropy in free quantum field theory, J. Phys. A 42 (2009) 504007 [arXiv:0905.2562] [SPIRES].

[4] T. Padmanabhan, Finite entanglement entropy from the zero-point-area of spacetime, [arXiv:1007.5066] [SPIRES].

[5] D. Nesterov and S.N. Solodukhin, Gravitational effective action and entanglement entropy in UV modified theories with and without Lorentz symmetry, arXiv:1007.1246 [SPIRES] .

[6] D.V. Vassilevich, Heat kernel expansion: User's manual, Phys. Rept. 388 (2003) 279 [hep-th/0306138] [SPIRES].

[7] T. Padmanabhan, Duality and zero-point length of spacetime, Phys. Rev. Lett. 78 (1997) 1854 [hep-th/9608182] [SPIRES]. 